\title{
Investigation on Fading of High Frequency Radio Signals Propagating in the Ionosphere - Results From a Jindalee Radar Experiment
}

\author{
K.S.B. Yau ${ }^{\dagger}$, C.J. Coleman ${ }^{\ddagger}$, and M.A. Cervera ${ }^{\S}$ \\ $\nmid$ School of Electrical and Electronic Engineering, The University of Adelaide, Australia. ksyau@eleceng.adelaide.edu.au \\ $\ddagger$ School of Electrical and Electronic Engineering, The University of Adelaide, Australia. \\ $\S$ Defence Science and Technology Organisation, Edinburgh, South Australia, Australia.
}

Keywords: HF Ionospheric Propagation, Signal Fading.

\begin{abstract}
High-Frequency (HF) radio-wave propagation in the ionosphere is still widely used. The ability to measure and understand the behaviour of the channel, and the knowledge of how the channel will affect the propagating signals, is imperative to ensure the reliability, and maintain adequate performance, of modern wide-bandwidth HF systems. An experiment to study the fading of HF signals propagating in the ionosphere has been conducted. Using the Jindalee Over-The-Horizon (OTH) radar, the behaviour of the ionospheric channel and wide bandwidth signal fading were observed. In this paper results from the experiment will be presented, and the potential uses for the set of experimental data will be discussed.
\end{abstract}

\section{Introduction}

The use of High-Frequency (HF) radio-wave propagation in the ionosphere is still prevalent for applications such as long-range communication, target detection and broadcasting. With the advance of data communication using $\mathrm{HF}$, and the introduction of HF digital radio broadcasting, there is a requirement that the ionospheric propagation channels must support a greater bandwidth. The ability to measure and understand the behaviour of the channel, and the knowledge of how the channel will affect the propagating signals, is imperative to ensure reliability, and maintain adequate performance, of modern wide-bandwidth HF systems. This is the motivation for conducting an experimental study of the fading of HF signals propagating in the ionosphere.

The experiment focusses on one of the most undesirable characteristics of HF signals, namely the variability of the signal levels arriving at the receiver, otherwise known as fading. The main contributors to the fading of HF signals propagating in the ionosphere are: polarisation, amplitude effects due to focussing and defocussing, and multi-path.

Polarisation fading occurs due to the rotation of the polarisation vector in a process known as Faraday rotation. Due to the bi-refringent nature of the ionosphere, a linearly polarised wave will split into two characteristic waves, the Ordinary $(\mathrm{O})$ and the Extraordinary $(\mathrm{X})$ which traverse the ionosphere with slightly different paths and speeds. Since they are polarised opposite in sense, the resultant wave arriving at the receiver will have a different polarisation compared to that of the initial wave. The dynamic nature of the ionosphere causes the polarisation of the wave at the receiver to change with time, and is manifest as fading in the received signal. Since polarisation fading is caused by the rotation of the electric field vector, the nature of this fading is that the received signal strength between two orthogonal antennas will be 90 degrees out of phase. Therefore signal maxima observed on one of the orthogonal antennas will correspond to signal minima on the other, and vice versa [4].

The movement of large-scale irregularities in the ionosphere is the cause for another fading mechanism: amplitude fading. Depending on the position of the irregularities, the ionosphere will effectively become a concave or convex mirror for HF radio-waves, which causes focussing or defocussing effects. Motion of the irregularity causes fading of the amplitude of the wave arriving at the receiver. The polarisation of the wave is unchanged, therefore the same amplitude fading effect will be observed on two orthogonal antennas. i.e. fading observed on orthogonal antennas will be in-phase.

Multi-path fading is caused by the presence of more than one propagation path between the transmitter and receiver. In contrast, the effects of polarisation fading and amplitude fading are apparent even where there is only a single propagation path. The waves from the different 
paths can interfere constructively or destructively when they arrive at the receiver depending on the phase difference. The phase difference between the different waves will vary in time due to the dynamic nature of the ionosphere, and therefore causes signal fading at the receiver. Multipath fading will produce a rapid variation in the signal amplitude, with the fading period in the order of several seconds, because it is highly sensitive to slight phase different between the paths. Conversely, focussing effects due to the movement of large-scale irregularities will produce a slower type of fading.

In the subsequent sections, descriptions of the fading experiment, along with the experimental results, will be presented.

\section{Experimental campaign}

The experimental campaign consisted of observing the fading of HF signals propagating through the ionosphere. The main objectives of the experiment were: (1) to characterise the fading of $\mathrm{HF}$ radio signals propagating in the ionosphere, and (2) to understand the different mechanisms that cause the different types of fading. In this paper, results on the characterisation of the different types of fading are presented. Ionosonde data recordings for the experimental period have recently become available, so objective (2) will also be achieved shortly and reported elsewhere.

The main experimental apparatus is the compact channel probe which was developed specifically for this experiment. Details on the channel probe and the suitable transmitter source will be given in the following section.

\subsection{Compact channel probe}

The compact channel probe was designed to include a number of important features: it is portable and easily reconfigurable, and is able to monitor horizontal and vertical polarisations simultaneously. A block diagram of the channel probe is shown in Figure 1.

To achieve portability, short-dipole active antennas were used. An active circuit performs the impedance matching [5], which enables good performance over the full $\mathrm{HF}$ region. Two dipole active antennas are arranged in the cross-dipole configuration to allow the monitoring of both vertical and horizontal polarisations.

The antennas were connected to a two-channel digital receiver. Software radio techniques [2] were used to develop a fully configurable channel probe, with tunable centre frequency and variable bandwidth. As the digital receiver card has two high-performance analog-to-digital convert-

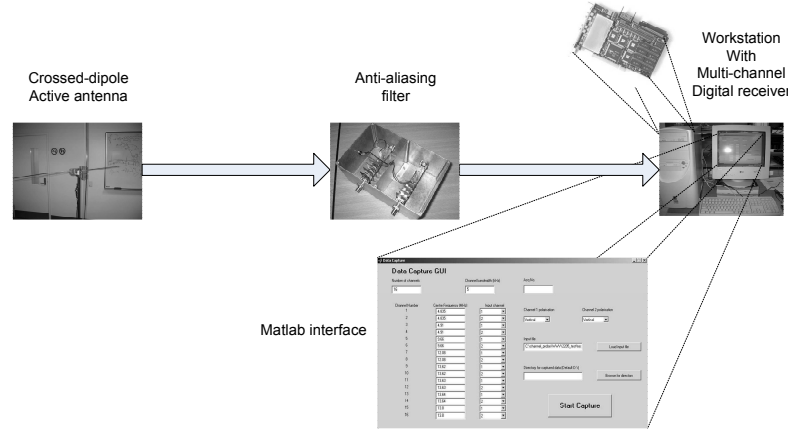

Figure 1: The compact Channel Probe

ers (ADC), and several digital down-converters, the full $\mathrm{HF}$ band can be digitised. To ensure that no signal aliasing was present after the ADC stage, a high performance low pass filter, with $80 \mathrm{~dB}$ attenuation at the Nyquist frequency of $32 \mathrm{MHz}$, was used. Configuration of the channel probe can be done in software via the MATLAB interface.

\section{$2.2 \quad$ Experimental details}

Data were collected over the three days 29th to 31st of March 2005. With the assistance of personnel from Defence Science and Technology Organisation, dedicated transmissions of HF radio signal for the sole purpose of this experiment were obtained from the Jindalee Over-theHorizon $(\mathrm{OTH})$ radar transmitter located in Alice Springs. The signal was received in Darwin which resulted in an ionospheric path $\sim 1220 \mathrm{~km}$ aligned almost in the northsouth direction, as shown in Figure 2.

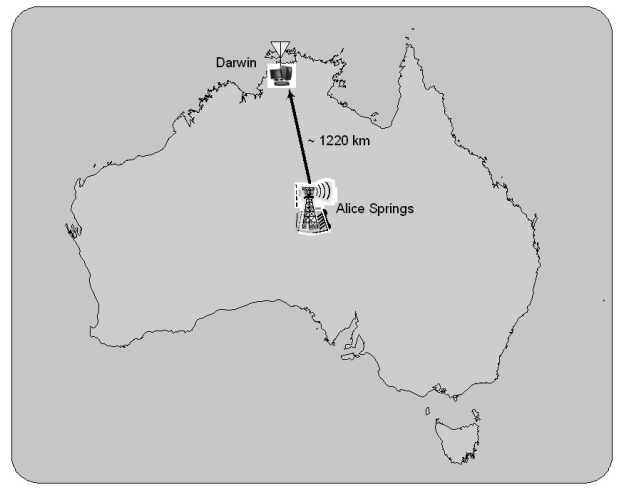

Figure 2: The $1220 \mathrm{~km}$ propagation path between Alice Springs and Darwin

Each day during the experiment, HF radio signals were transmitted from 15:00 until 21:00 local time (LT). The centre frequency and bandwidth of the signals were based on the spectral availability at the time of transmission, determined by the frequency management system (FMS) of the Jindalee OTH radar [3]. On the 30th of March, two bands of frequency were available from around 16:00 until 21:00 LT viz. 10.677 MHz and $10.858 \mathrm{MHz}$, with band- 
widths of $46 \mathrm{kHz}$ and $48 \mathrm{kHz}$ respectively. The transmitted signals were Frequency-Modulated Continuous-Wave (FMCW) with a full-bandwidth sweep repetition rate of $5 \mathrm{~Hz}$.

Unfortunately the radar was not set up optimally. The geographic footprint of the transmit beam cycled between adjacent regions every 14 seconds. Thus, the signal level was observed to vary in a step fashion with a 14 -second period. To overcome this problem, the data were power compensated with an empirically calculated factor.

\section{Results}

Our analysis concentrated on the data collected on the 30th of March 2005, because of its completeness. Using the FMCW processing techniques [1], and synthesised version of the transmitted FMCW waveform, the received chirp signal can be demodulated down to baseband. The various receive mode time-delays are translated into different frequency-offset bins, and thus can be separated (different propagation modes will have greatly different time delays). The Kaiser window was used in the signal processing to suppress the side-lobes and thus ensure the absence of false modes [6].

The spectrogram of the received modes in the $10.677 \mathrm{MHz}$ band at 18:42 LT (local sunset period) is shown in Figure 3. Multiple propagation modes are clearly present at this time. The presence of the different modes indicates that multi-path fading will probably dominate during this time.

In this paper, time fading is defined as the signal strength variation in the temporal sense, and frequency fading is defined as the signal strength variation across the frequency bandwidth. The time and frequency fading behaviour of the combined modes are shown in Figure 4 (a) and (b) respectively. With the short time-period and the

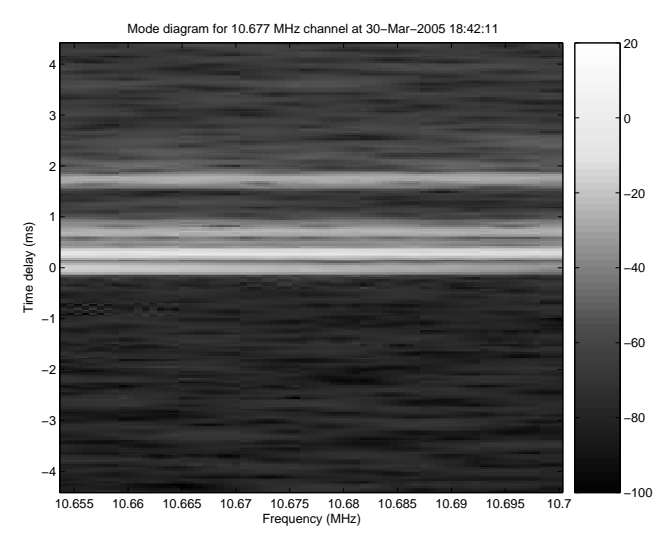

Figure 3: Mode spectrograms of the $10.677 \mathrm{MHz}$ band commencing at 18:42 LT on the 30th March 2005.

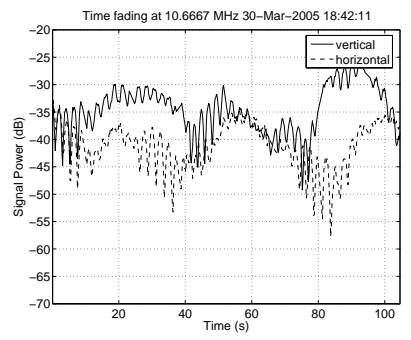

(a)

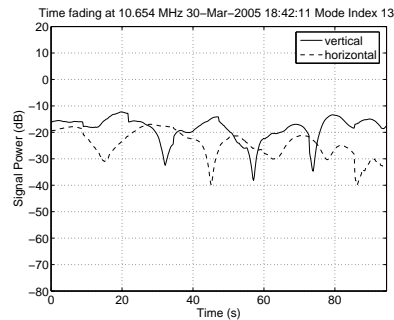

(c)

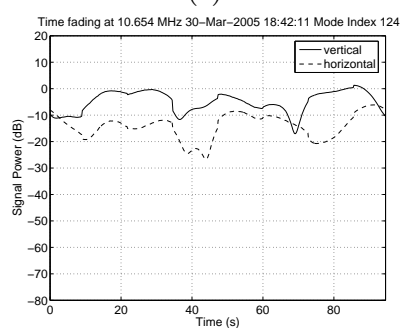

(e)

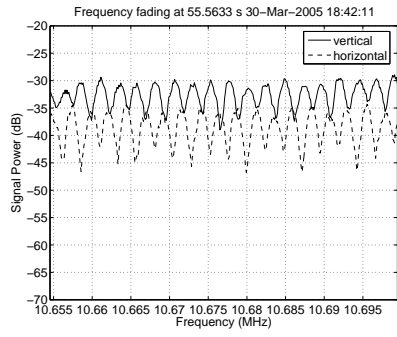

(b)

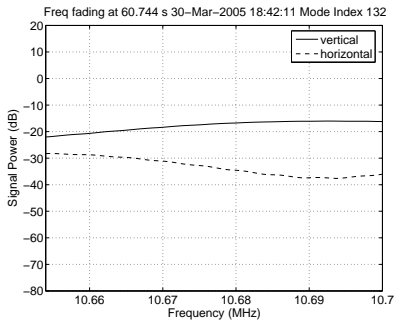

(d)

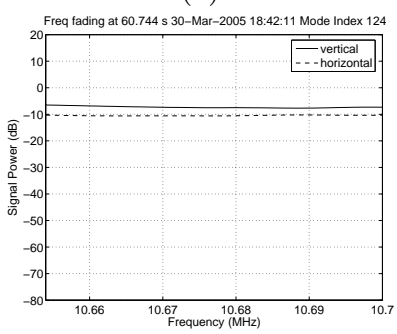

(f)
Figure 4: Fading behaviour of the signal commencing at 18:42 LT for vertical (solid-line) and horizontal (dash-line) polarisations. (a) Multi-path time fading. (b) Multi-path frequency fading. (c) Time fading for the first received mode. (d) Frequency fading for the first received mode. (e) Time fading for the second received mode. (f) Frequency fading for the second received mode.

almost periodic sinusoidal nature of time fading, this has the signature of multi-path fading due to two or more different propagation modes. Examination of the frequency fading behaviour shows that the fading was not flat across the frequency bandwidth; the signal level changes quite rapidly. This has important implications for modern highbandwidth HF systems i.e. the assumption of flat fading across the available frequency bandwidth is not valid.

Utilising FMCW processing techniques, it is possible to determine the fading behaviour of individual modes. Time and frequency fading behaviour of the first received mode is shown in Figure 4 (c) and (d) respectively. It can be seen that the fading between the two antennas is about 90 degrees out of phase, which indicates the fading mechanism is polarisation fading. This is an important observation because polarisation fading becomes the dominant fading mechanism for each individual mode. Looking at the frequency fading behaviour, the out of phase fading between the two antennas is still apparent. However the signal bandwidth is not wide enough to observe the full frequency fading bandwidth which might be due to polarisation fading. Similar behaviour is shown in Figure 4 
(e) and (f) for the second mode. This is consistent with simulation results for polarisation fading [7].

Figure 5 displays the spectrogram of the received modes in the $10.677 \mathrm{MHz}$ band at 19:45 LT which is well after sunset. It is clear that only a single mode was present, and the time and frequency fading behaviour of this mode are shown in Figure 6 . The signature of polarisation fading is apparent but at a slower rate compared to polarisation fading during sunset. This confirms that polarisation fading is the dominant signal fading mechanism for a single mode propagation path.

We now examine the extended time fading behaviour of the received mode in the $10.677 \mathrm{MHz}$ band commencing at 19:25 LT in Figure 7. In addition to the polarisation fading signature, there is also a slower variation in the amplitude of the signal peaks. This slower variation was observed in both polarisations, and is the signature of amplitude fading.

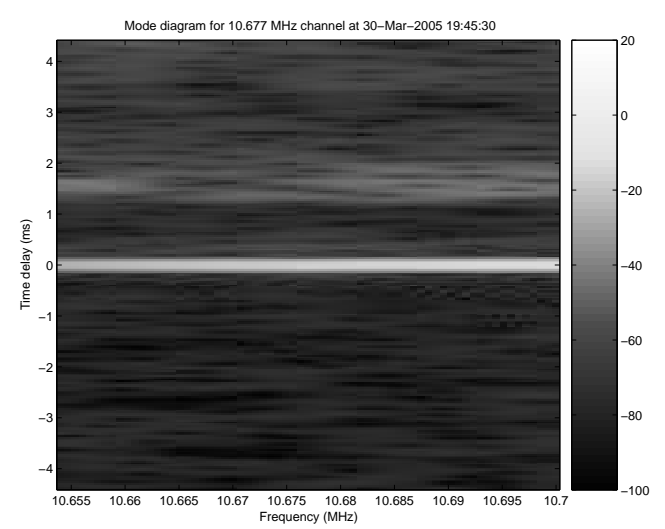

Figure 5: Mode spectrograms of the $10.677 \mathrm{MHz}$ band commencing at 19:45 LT on the 30th March 2005.

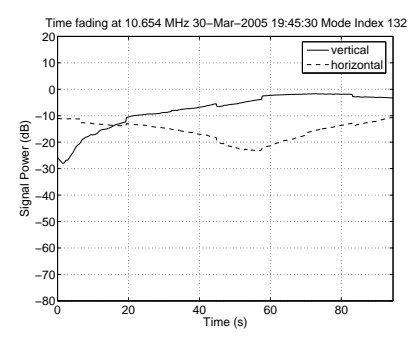

(a)

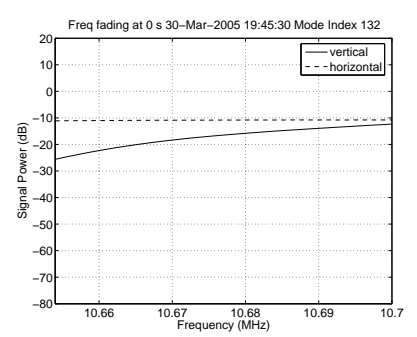

(b)
Figure 6: Fading behaviour of the signal commencing at 19:45 LT for vertical (solid-line) and horizontal (dash-line) polarisations. (a) Time fading behaviour of the single mode. (b) Frequency behaviour of the single mode.

\subsection{Fading separation}

Amplitude and polarisation fading may be separated by simple data manipulation. The received voltages at the two orthogonal antennas are given by:

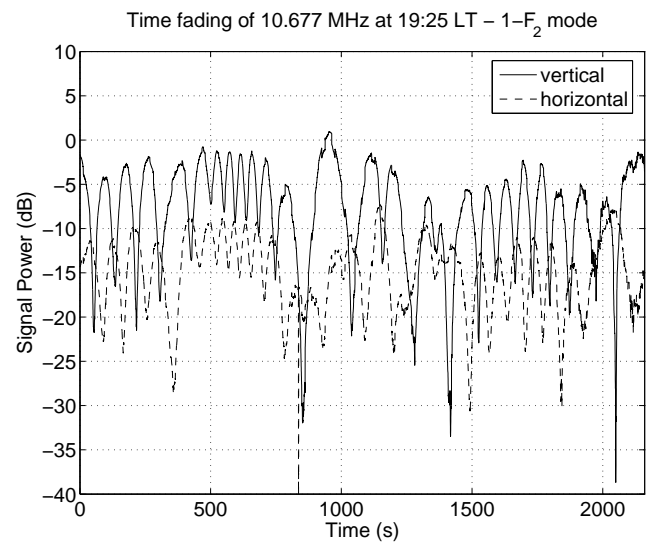

Figure 7: Extended time fading behaviour of $10.677 \mathrm{MHz}$ signal commencing at 19:25 LT for vertical (solid-line) and horizontal (dash-line) polarisations.

$$
\begin{aligned}
& V_{H}=A(f, t) \cos (\phi(f, t)) \\
& V_{V}=k A(f, t) \sin (\phi(f, t))
\end{aligned}
$$

where $A(f, t)$ is the amplitude component of the received signal, $\phi(f, t)$ is the phase component of the received signal, and $k$ is the constant amplitude offset between the two antennas. The constant amplitude offset factor $k$ was estimated by taking the ratio of long time average of voltage amplitudes $V_{H}$ and $V_{V}: k=\frac{\overline{\left|V_{V}\right|}}{\left|V_{H}\right|}$.

Rearranging (1) and (2) gives the following for the phase and amplitude components:

$$
\begin{aligned}
\tan \phi & =\frac{V_{V}}{k V_{H}} \\
A^{2} & =V_{H}^{2}\left(1+\tan ^{2} \phi\right)
\end{aligned}
$$

Using (3) and (4) one can determine the level of contribution of polarisation and amplitude fading on the overall fading of the signal. If the amplitude fading component given by (4) is varying rapidly, whereas the phase component given by (3) is steady, then amplitude fading dominates. Conversely, if the amplitude component is constant, while the phase varies then polarisation fading dominates.

The results of fading separation for the first received mode in the 10.677 MHz band at 18:42 LT are shown in Figure 8. The amplitude fading has a periodicity in the order of several tens of seconds. The polarisation fading factor, which is the amplitude variation due to polarisation fading, also shows a period of the same order. This reasonably fast fading is most likely caused by the rapid variation in the electron density during the sunset period.

Figure 9 shows the fading separation results for the period after sunset. Both the amplitude fading and polarisation 
fading factors are much slower, as expected for a more benign ionosphere compared to the sunset period. The jumps in the amplitude fading power are attributed to the power compensation that was used to overcome the effects of the moving radar beam. The amplitude fading separation was able to detect the power compensation because of the over-compensation applied to the received signal from both horizontal and vertical antennas.

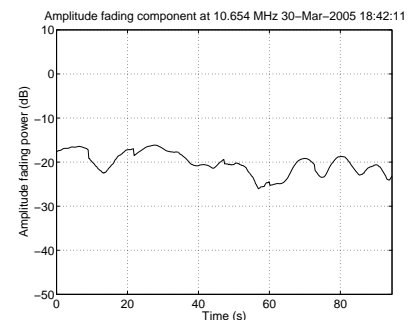

(a)

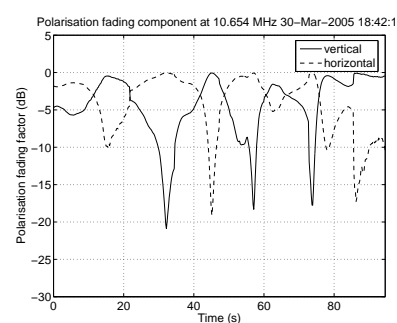

(b)
Figure 8: Fading separation of first received mode commencing at 18:42 LT. (a) Amplitude fading power. (b) Polarisation fading factor which shows the variations in amplitude due to polarisation fading, for for vertical (solid-line) and horizontal (dash-line) polarisations.

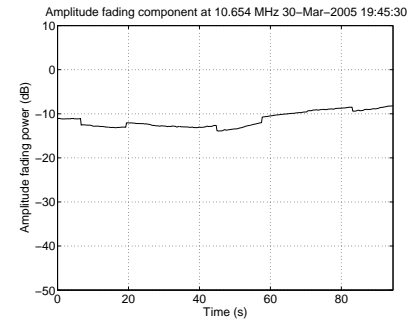

(a)

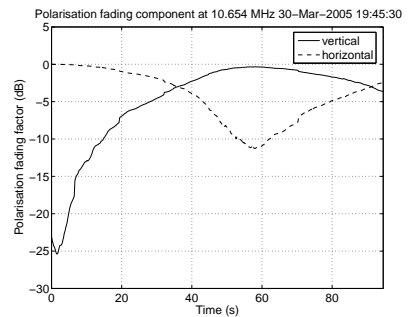

(b)
Figure 9: Fading separation of first received mode commencing at 19:45 LT. (a) Amplitude fading component. (b) Polarisation fading factor which shows the variations in amplitude due to polarisation fading, for vertical (solid-line) and horizontal (dash-line) polarisations.

\section{Discussion and future work}

There are a number of conclusions from this experiment. Firstly, during the period when multi-path is prevalent, there is significant signal fading in frequency and consequently the assumption of flat-fading across bandwidth becomes invalid. Another observation is that polarisation fading is always present but can be masked by multi-path fading. Finally, the results confirm that rapid signal fading is associated with the period when the ionosphere is undergoing rapid changes.

Comparisons between the signal fading data and ionosonde data are well underway and it is hopeful that results will soon become available. In doing this comparison, one will be able to discover the exact structure of the ionosphere during the period of rapid fading, thus identifying the types of irregularity that are causing the different types of fading. Furthermore, the ionosonde data enables a more realistic modelling of the fading.

When fully analysed, the data set will become an important tool for studying the types of fading that one can expect for various ionospheric conditions. The data could also be used to test various mitigation techniques to combat signal fading, such as polarisation diversity and frequency diversity. Also it is of interest to analyse the effects of signal fading on HF Multiple-Input MultipleOutput (MIMO) systems, and whether, through MIMO, HF multi-paths can be put to advantage.

\section{Acknowledgements}

We thank the Defence Science and Technology Organisation for providing its valuable radar resources for use during this experiment.

\section{References}

[1] D. E. Barrick. FM/CW radar signals and digital processing, Technical Report ERF 283-WPL 26, NOAA, July 1973.

[2] N. C. Davies. A high performance HF software radio, Eighth International conference on HF Radio Systems and Techniques, Conference pub. 474, pp. 249-256. IEE, 2000.

[3] G. F. Earl and B. D. Ward. The frequency management system of the Jindalee over-the-horizon backscatter HF radar, Radio Science, 22(2):275-291, MarchApril 1987.

[4] D. A. Hedlund and L. C. Edwards. Polarization fading over an oblique incidence path, IRE Transaction on Antennas and Propagation, 6(1):21-25, January 1958.

[5] M. J. Salvati. A miniature broadband antenna, Electronic Design, February 1995.

[6] E. M. Warrington. On the processing of FMCW signals for ionospheric sounding applications, $H F R a-$ dio Systems and Techniques, Conference pub. 392, pp. 379-382. IEE, July 1994.

[7] K. S. B. Yau. A simple polarisation fading model for HF propagation in the ionosphere, Ninth International conference on HF Radio Systems and Techniques, Conference pub. 493, pp. 131-135. IEE, June 2003. 